\title{
Time for a new definition of Parkinson's disease
}

\author{
Wolfgang H. Jost ${ }^{1}\left[\right.$ ] Heinz Reichmann ${ }^{2}$
}

Received: 21 December 2018 / Accepted: 27 March 2019 / Published online: 5 April 2019

(c) Springer-Verlag GmbH Austria, part of Springer Nature 2019

Parkinson's disease is traditionally defined as a motor disease characterized by the triad of akinesia, rigidity and tremor, whereby bradykinesia is the obligatory symptom for a secure diagnosis. However, in the past 20 years, the non-motor symptoms (NMS) have gained increased attention, although they are still considered "lesser lights" compared to the motor ones, and are still only rarely considered in formulating diagnostic criteria. But importantly: most of the NMS are in fact more frequent than, for example, resting tremor and more severely restrict quality of life through the course of the disease due to the fact that their therapeutic treatment is limited.

New findings share a common aspect with clinic expansions: new buildings in a hospital always signal problems in orientation for both patients and clinic personnel. Not infrequently, a new and easily navigable building would be called for, in which architecture fits the requirements. And that is precisely the case with NMS: we now need a classification according to the clinical picture instead of second-order symptoms. And in our view, the time is now ripe for such an endeavor.

We should now classify just which NMS belong to the disease and at which stage of the disease they occur, that is, prior to, concomitant with or subsequent to the motor signs. Then, we should define just which of these NMS is a direct or an indirect consequence of Parkinson-specific neurodegeneration or rather of the pharmacological therapy, and then exclude NMS which are present due to some other disease. It is also crucial to understand to what extent the various symptoms correlate with each other as to their occurrence, their degree of severity and their fluctuations. We also have to critically ask how much diagnostic relevance the individual markers really possess, for example,

Wolfgang H. Jost

w.jost@parkinson-klinik.de

Heinz Reichmann

Heinz.Reichmann@uniklinikum-dresden.de

Parkinson-Klinik Ortenau, Wolfach, Germany

2 Department of Neurology, Universitätsklinikum Carl Gustav Carus, Dresden, Germany whether REM sleep disturbance has predictive merits and is sufficiently sensitive and specific.

But it may be even more important that the NMS are no longer interpreted as being merely attendant symptoms. Only in this way can we do justice to our patients and also make progress at the same time in understanding the pathogenesis of the disease. We also have to work on giving more attention to diagnostics and therapy of NMS. This is already successfully the case for neuropsychiatric symptoms and sleep disturbances, but there is considerably more to be done for autonomic disturbances, with the result that other medical fields work on the basics of diagnosis and therapy.

For this reason, we chose NMS as our main area of focus for the 19th Experts Meeting on Parkinson's. Daniela Berg (Kiel) summarized the current status of biomarkers for the early diagnosis of Parkinson's disease in which NMS is clinically predominantly present. Anette Schrag (London) concentrated on the neuropsychiatric disturbances of idiopathic Parkinson's syndrome (IPS), and Jaime Kulisevsky (Barcelona) elaborated on the early cognitive disturbances in IPS. Christian Baumann (Zurich) treated specific sleep disturbances uniquely relevant for both diagnostics and therapy. To date, the non-motor fluctuations have received far too less attention. Their cause and differentiation from motor fluctuations were the subject of the contribution from Alexander Storch (Rostock). Our main area of focus was extended with the importance of cannabinoids for the treatment of IPS as well as workshops on the topic of managing delirium in IPS, motor fluctuations in atypical Parkinson's syndrome, polypharmacy in IPS and the relevancy of alpha-synuclein in the pathogenesis of Parkinson's disease.

Finally, we should redefine the concept of Parkinson's disease, a neurodegenerative disease that affects both the peripheral and the central nervous system with symptoms that have different onsets and courses of progression. Neither oversimplifying the symptoms nor a rigid adherence to older models are of service anymore for furthering our understanding of the disease which is inherently a complex one, but precisely that's what makes it so intriguing.

Publisher's Note Springer Nature remains neutral with regard to jurisdictional claims in published maps and institutional affiliations. 